\title{
Measurement Methods for Train Localization with Onboard Sensors
}

Oliver Heirich
Benjamin

Siebler
Stephan

Sand
Andreas

Lehner
Omar

García Crespillo

$$
\text { DLR - German Aerospace Center }
$$

Institute of Communications and Navigation, Oberpfaffenhofen, Germany

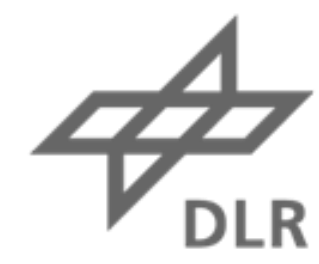




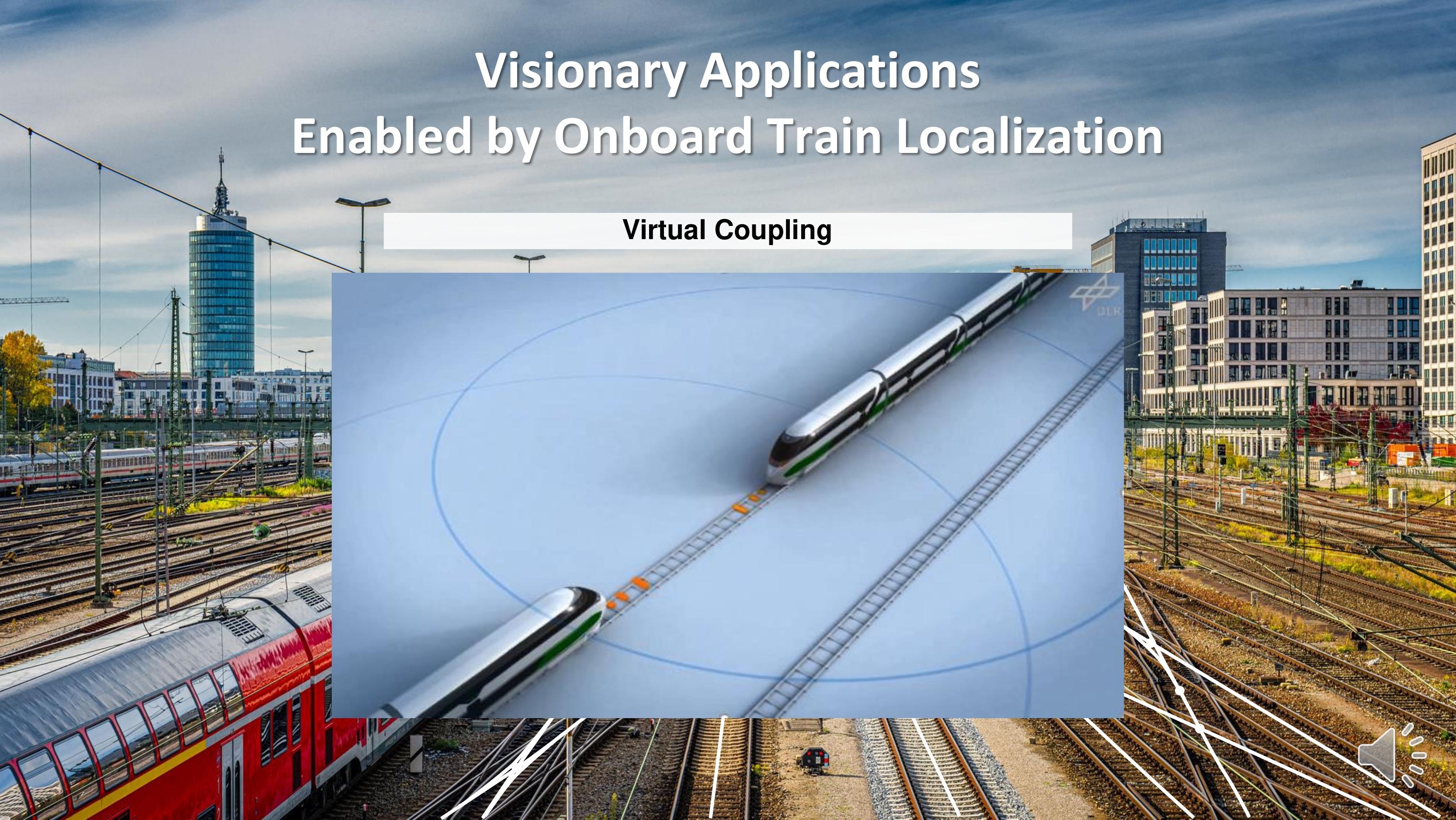




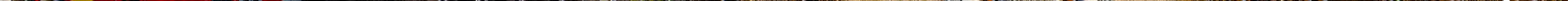




\section{Applications Enabled by Onboard Train Localization}

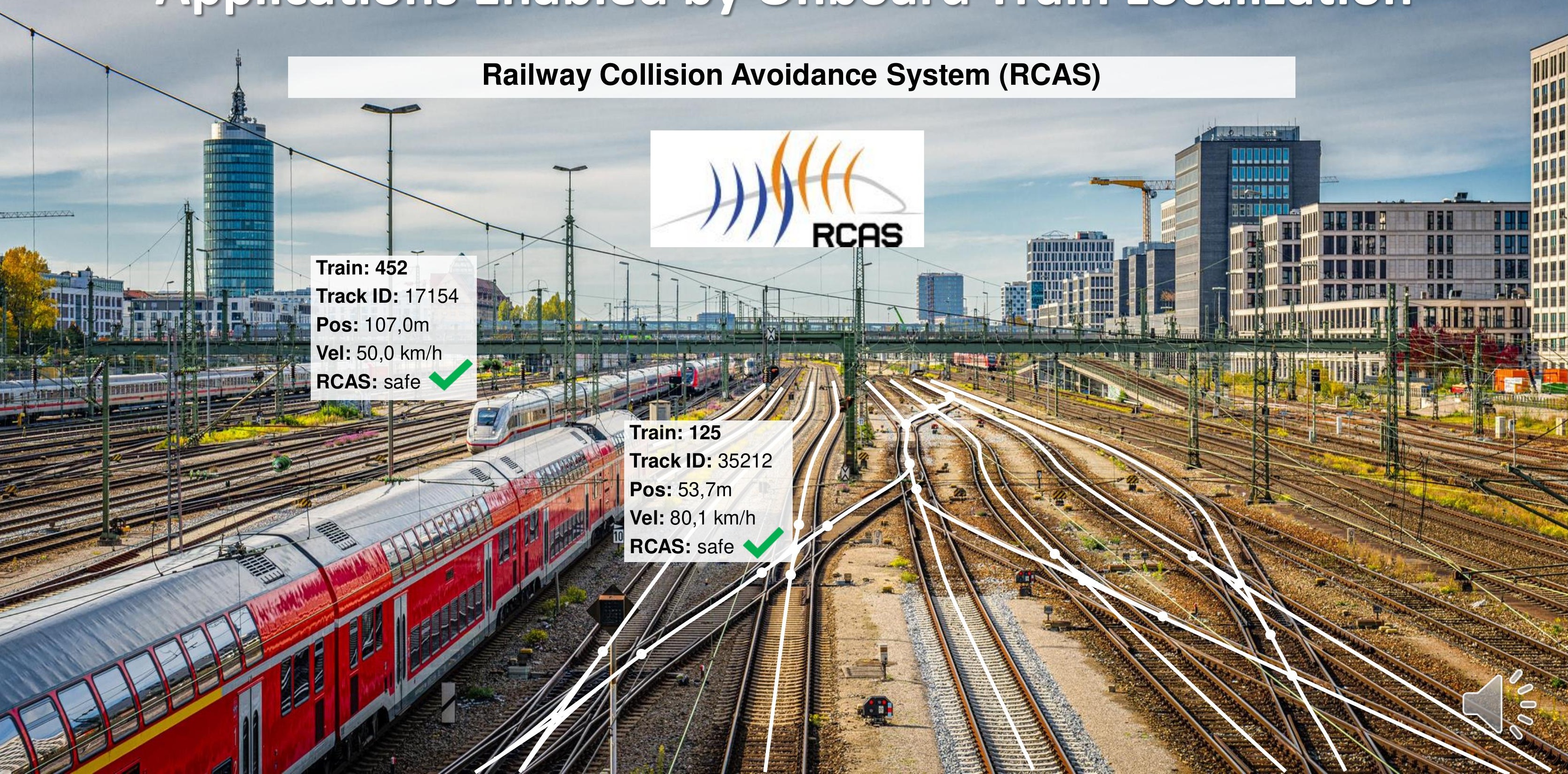




\section{Applications Enabled by Onboard Train Localization}

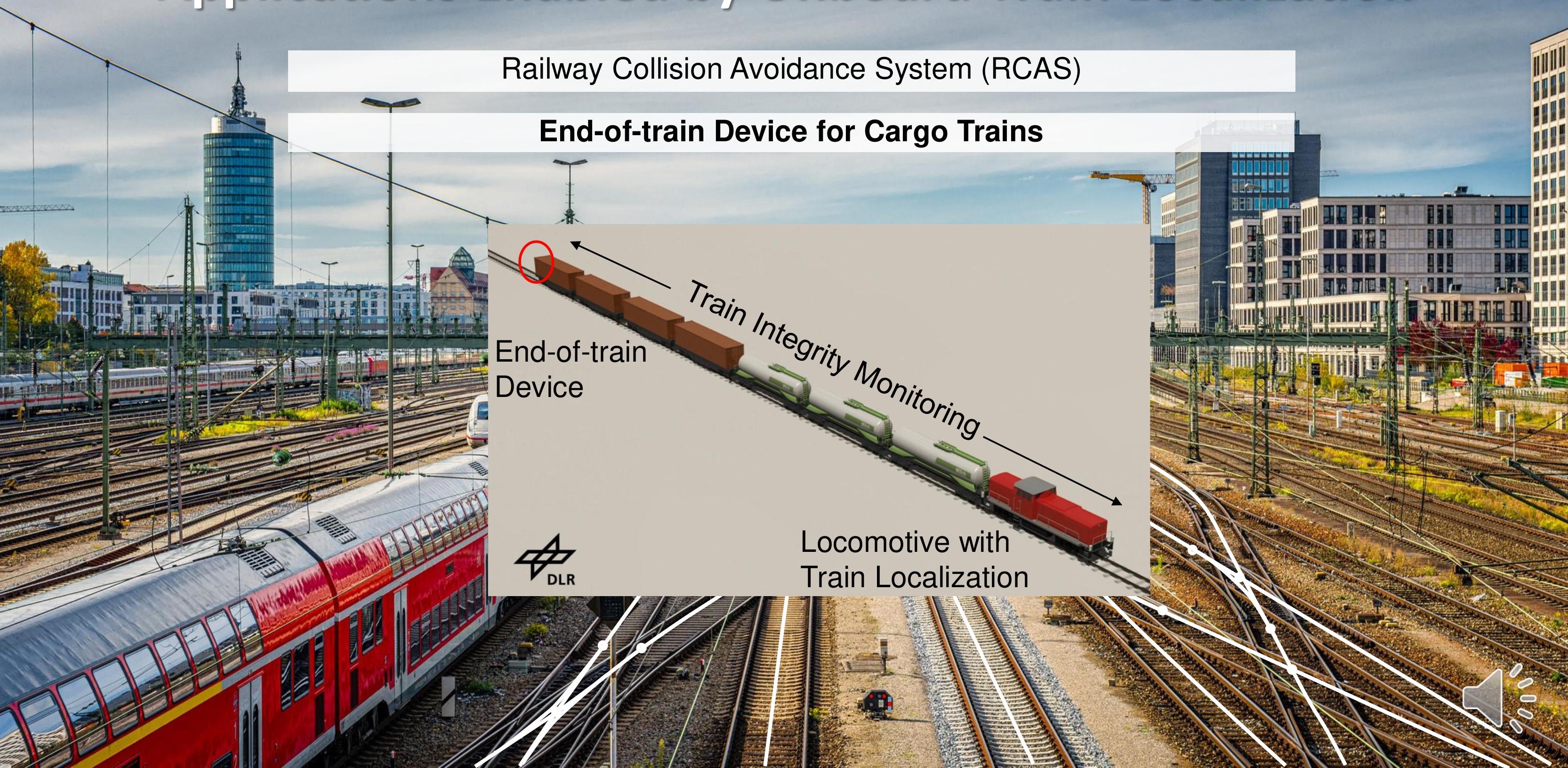




\section{Applications Enabled by Onboard Train Localization}

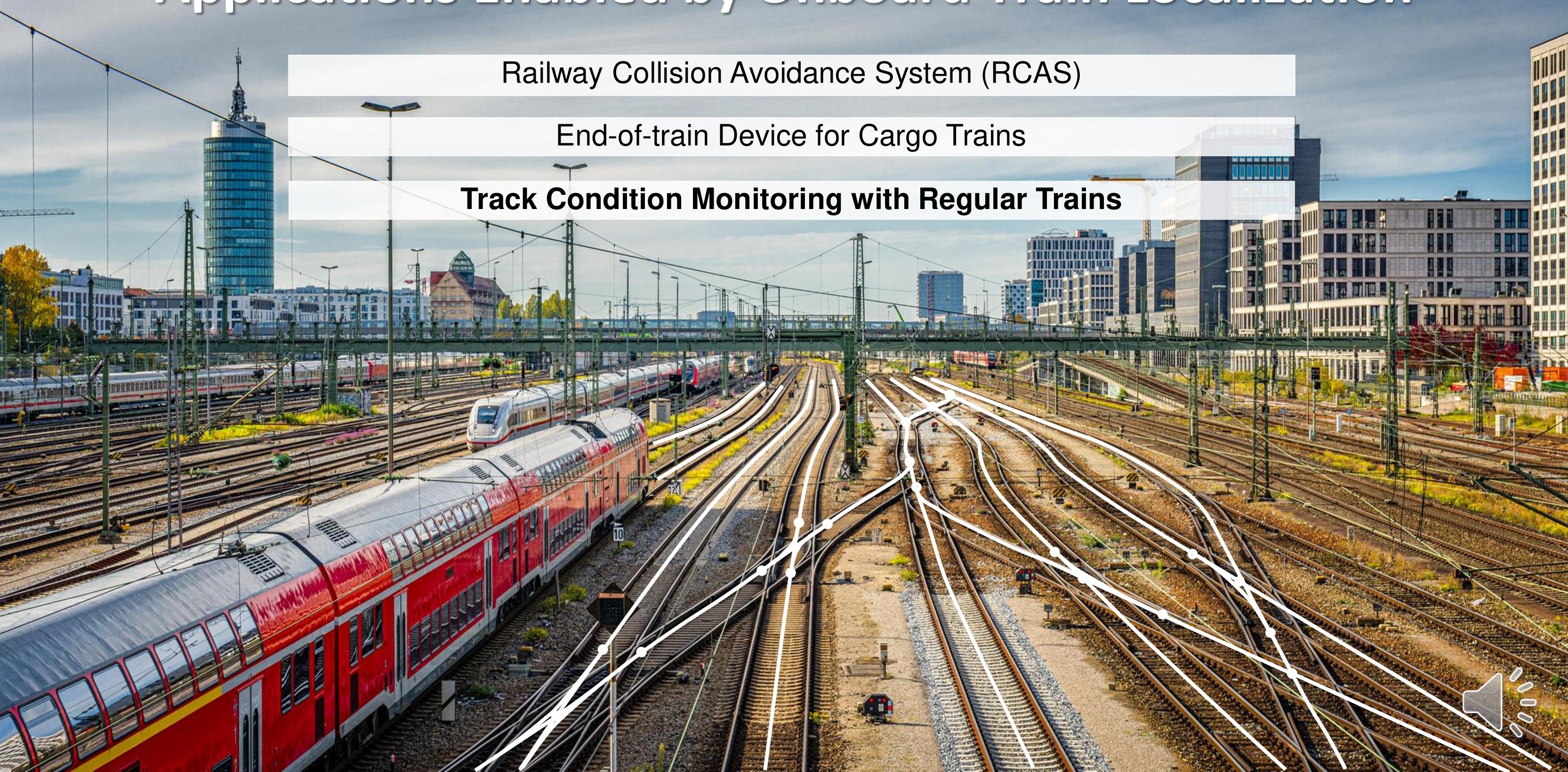




\section{Applications Enabled by Onboard Train Localization}

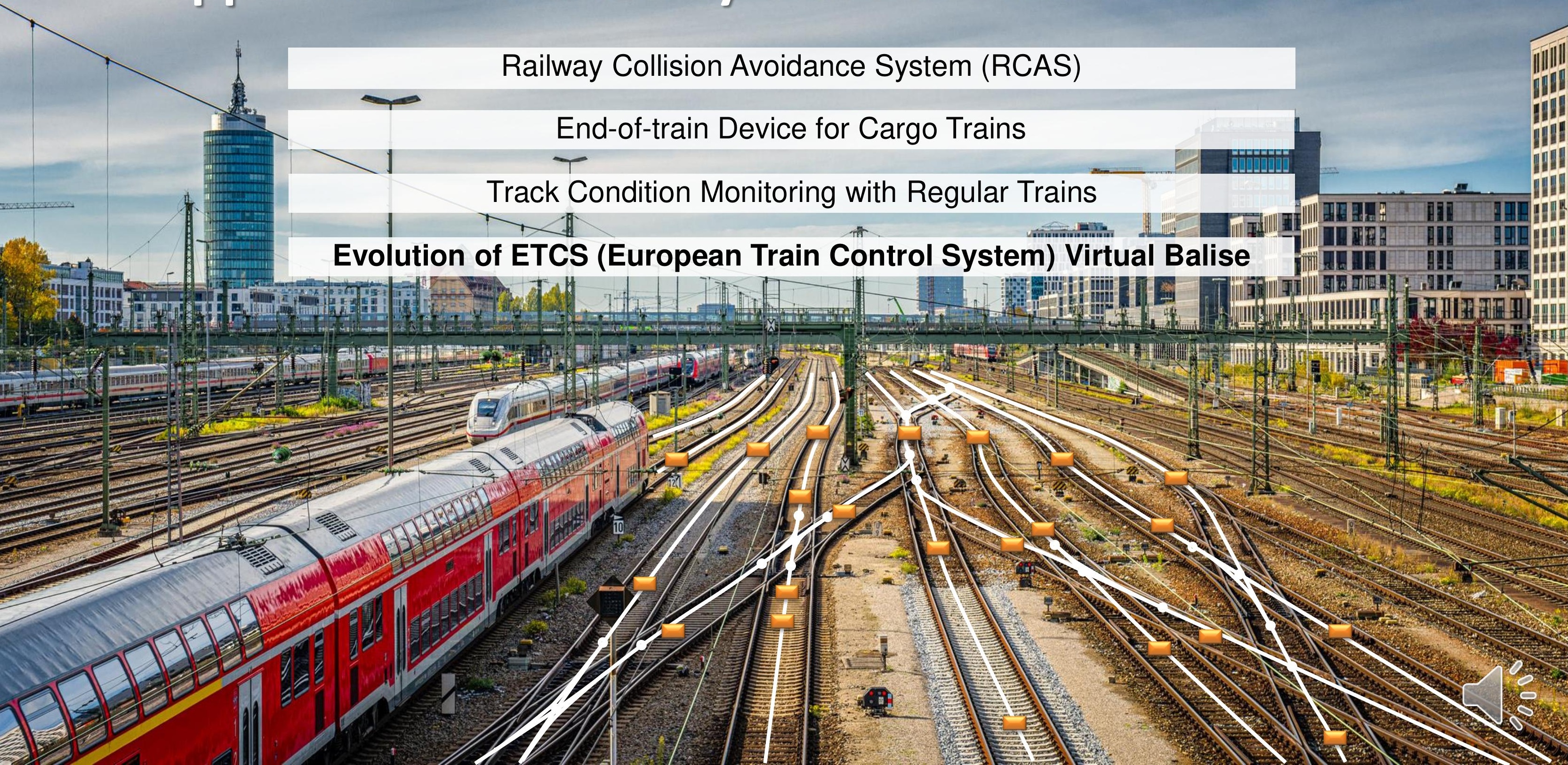




\section{Onboard Train Localization}

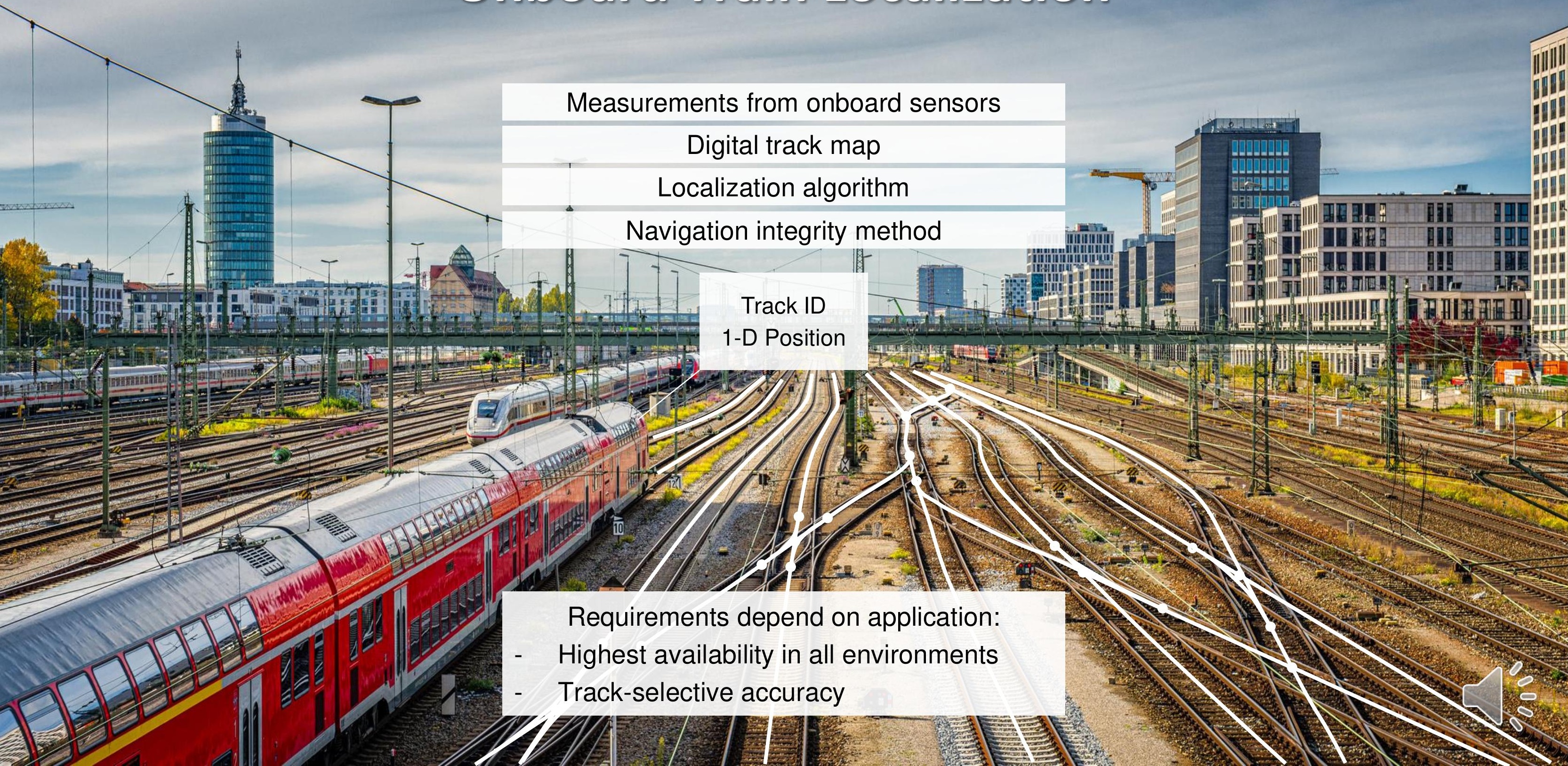




\section{Onboard Train Localization}

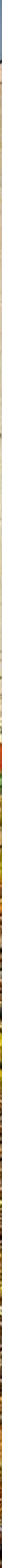




\section{How can a Measurement Contribute to Train Localization?}

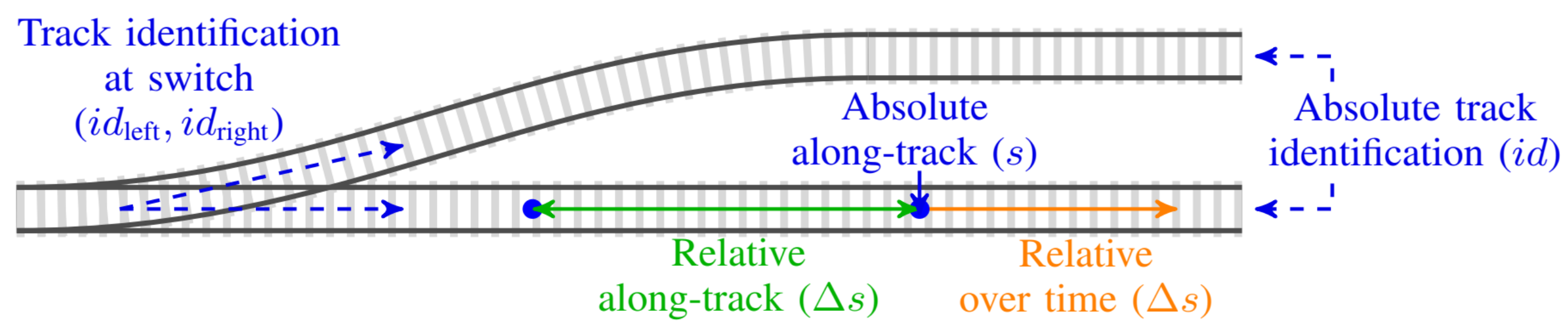

\begin{tabular}{|l|c|}
\hline Train Speed, Relative over Time \\
\hline Speed measurement & $\mathrm{d} / \mathrm{dt} s$ \\
\hline Travel distance over time & $\Delta s$ \\
\hline Dead reckoning & $\Sigma \Delta s$ \\
\hline
\end{tabular}

\section{Relative Train Localization} Distance measurement between $\Delta s$ two positions on track

\section{Absolute Train Localization} Absolute along-track

Absolute track identification

Track identification at switches

$s$

id

id

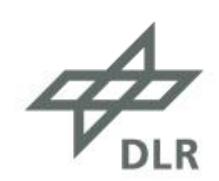




\section{Absolute and Relative Train Localization}

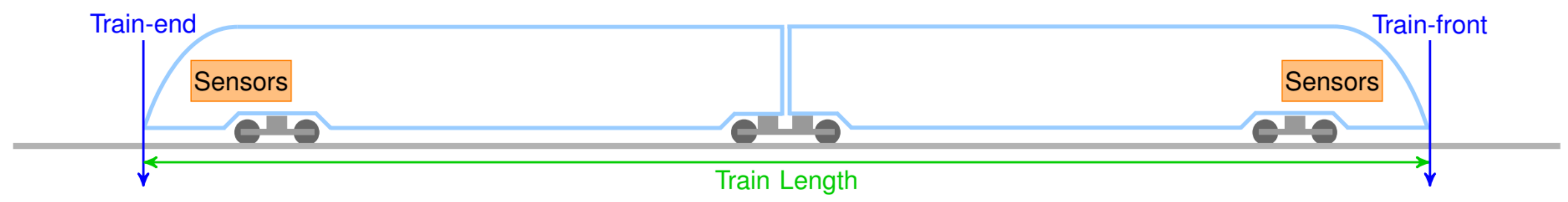

Absolute Train Localization

Relative Train Localization

Train length

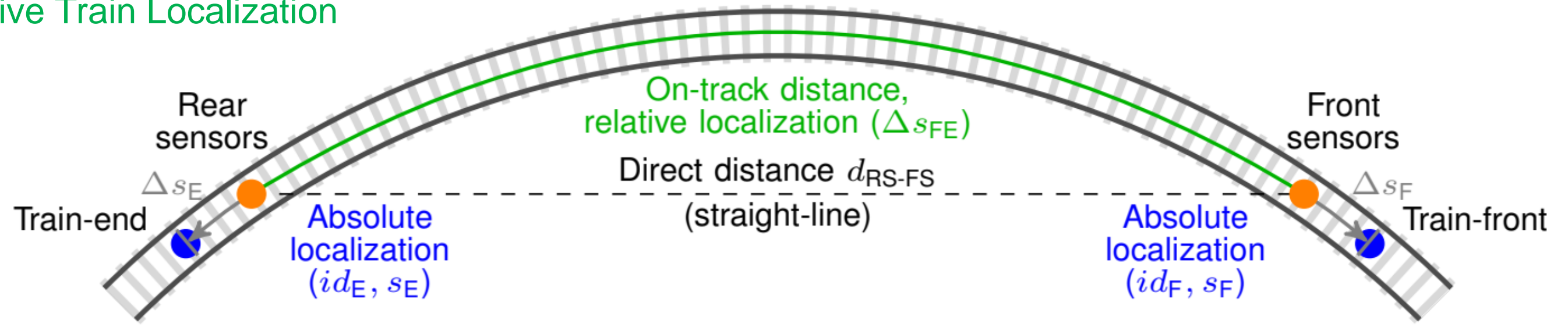




\section{Robust and Safe Train Localization}

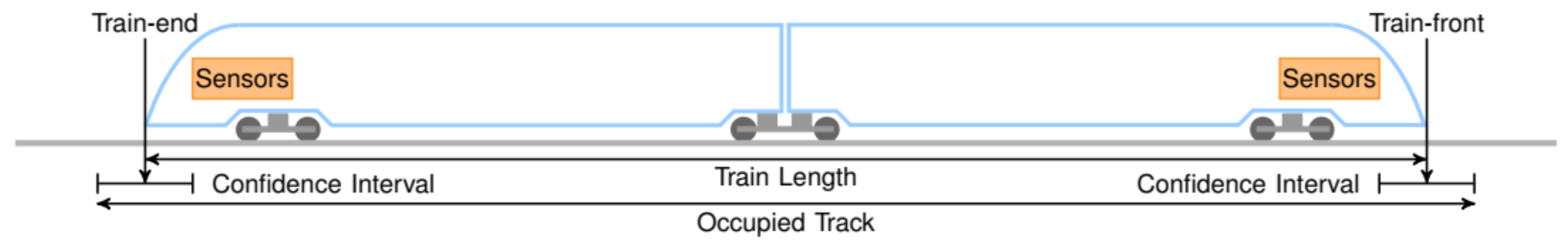

- Safe train localization uses confidence intervals and a defines an occupied track

- redundant measurements

- navigation integrity monitoring

- Navigation integrity method computes protection levels based on measurement analysis, failure detection and exclusion and a sensor fusion monitoring

- Confidence interval is the protection level in along track direction

- Navigation integrity guarantees, that true location is within the confidence interval with very low error probability

- An application is safe when the confidence interval (protection level) is smaller than a limit (alert limit) that is defined by the application and use-case requirements 


\section{Measurement Methods Overview}

- Sensors (selection)

1. GNSS,

2. IMU,

3. Magnetometer,

4. Terrestrial radio ranging

- Some methods require a map, pre-processing, or multiple measurements

- Paper contains further links to publications

\begin{tabular}{|c|c|c|c|}
\hline & Type & Method & Sensor \\
\hline S.1 & GNSS & Doppler, time differenced carrier phase & GNSS receiver \\
\hline S.2 & Magnetic & periodic wheel signal & magnetometer \\
\hline S.3 & Magnetic & speed signature & magnetometer \\
\hline S. 4 & Magnetic & magnetic signature & $2 \mathrm{x}$ magnetometer \\
\hline S.5 & Vibration & speed signature & inertial \\
\hline S.6 & Vibration & vibration signature & $2 \mathrm{x}$ inertial \\
\hline S.7 & Cellular RF system & Doppler, base station to mobile & mobile terminal \\
\hline
\end{tabular}

\section{Relative Train Localization}

\begin{tabular}{|c|c|c|c|}
\hline & Measurement Method & Type of Localization & Sensors, Map \\
\hline R.1 & GNSS relative & $\begin{array}{l}\text { relative on tracks, } \\
\text { train to train / front to end }\end{array}$ & $2 x$ GNSS (SBAS), map \\
\hline R.2 & Baseline GNSS & relative, direct distance & $2 x$ GNSS \\
\hline R.3 & INS relative & relative on tracks & IMU, (GNSS,X,odometer,map) \\
\hline R.4 & Inertial signature & relative on tracks & 2x IMU, (odometry) \\
\hline R.5 & Magnetic signature & relative on tracks & $2 \mathrm{x}$ magnetometer, (odometry) \\
\hline R.6 & Radio ranging on train & $\begin{array}{l}\text { relative, direct distance } \\
\text { train to train / front to end }\end{array}$ & $2 \times \mathrm{RF}$ tranceivers \\
\hline R.7 & Cellular base station & $\begin{array}{l}\text { relative, direct distance } \\
\text { train to train / front to end }\end{array}$ & $2 \mathrm{x}$ cellular mobile terminal, map \\
\hline
\end{tabular}

\section{Absolute Train Localization}

\begin{tabular}{|c|c|c|c|}
\hline & Measurement Method & Type of Localization & Sensors, Map \\
\hline A. 1 & GNSS loosely & $\begin{array}{l}\text { absolute on tracks, } \\
\text { loosely coupled with map }\end{array}$ & GNSS position, (SBAS), map \\
\hline A. 2 & GNSS tightly & $\begin{array}{l}\text { absolute on tracks, } \\
\text { tightly coupled with map }\end{array}$ & GNSS pseudorange, (SBAS) map \\
\hline A. 3 & INS/GNSS & absolute on tracks & IMU, GNSS, map \\
\hline A. 4 & $\mathrm{INS} / \mathrm{X} / \mathrm{map}$ & absolute on tracks & $\begin{array}{l}\text { IMU, (odometer), map } \\
\text { X: e.g. magnetom., vibrations }\end{array}$ \\
\hline A. 5 & Inertial signature & absolute on tracks & IMU, (odometry), map \\
\hline A. 6 & Magnetic signature & absolute on tracks & magnetometer, (odometry), map \\
\hline A.7 & $\begin{array}{l}\text { Cellular base station } \\
\text { radio ranging }\end{array}$ & $\begin{array}{l}\text { absolute on tracks, } \\
\text { loosely coupled with map }\end{array}$ & cellular mobile terminal, map \\
\hline A. 8 & $\begin{array}{l}\text { Cellular base station } \\
\text { radio ranging }\end{array}$ & $\begin{array}{l}\text { absolute on tracks, } \\
\text { tightly coupled with map }\end{array}$ & cellular mobile terminal, map \\
\hline
\end{tabular}




\section{GNSS - Global Navigation Satellite System}

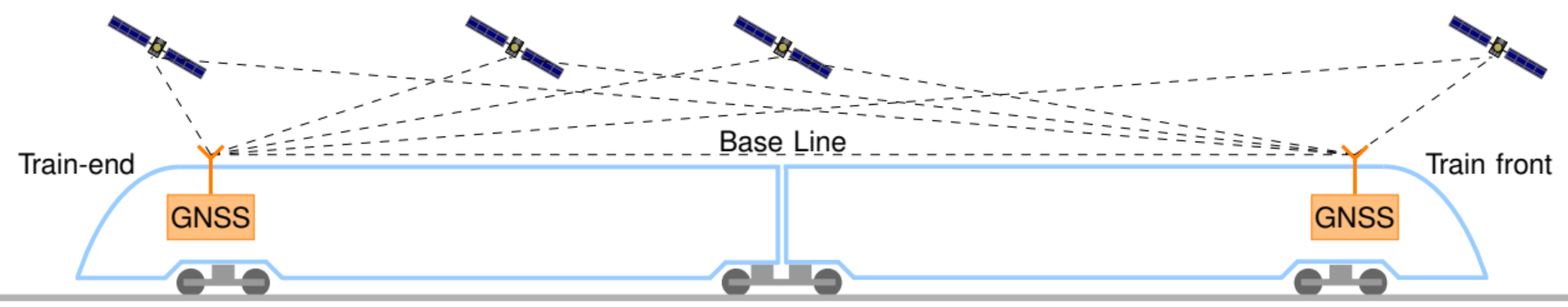

A GNSS receiver measures pseudorange, Doppler and carrier phase to multiple satellites in view.

\begin{tabular}{|c|c|}
\hline d, Rele & - Tims \\
\hline Train speed via Doppler & GNSS \\
\hline $\begin{array}{l}\text { rain speed via time } \\
\text { arrier phase }\end{array}$ & \\
\hline
\end{tabular}

\begin{tabular}{|c|c|}
\hline \multicolumn{2}{|c|}{ Relative Train Localization } \\
\hline Relative on tracks & 2x GNSS \\
\hline $\begin{array}{l}\text { Baseline GNSS, double } \\
\text { differencing }\end{array}$ & \\
\hline
\end{tabular}

\begin{tabular}{l}
\begin{tabular}{l} 
Absolute Train Localization \\
\hline $\begin{array}{l}\text { GNSS position, loosely } \\
\text { coupled with map }\end{array}$ \\
$\begin{array}{l}\text { GNSS pseudorange, tightly } \\
\text { coupled with map }\end{array}$
\end{tabular} \\
\hline
\end{tabular}

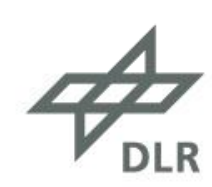




\section{IMU - Inertial Measurement Unit}

Measurements: accelerations, turn rates in three axes $(x, y, z)$

\section{Inertial Navigation System (INS/GNSS, INS/X/map):}

- INS/GNSS computes position, velocity and attitude

\section{Kinematics of track geometry}

- No integration required: suitable for low-cost MEMS IMUs

- Curvatures can be processed from turn rates and velocity

\section{Vibrations}

- Characteristic vibration intensity

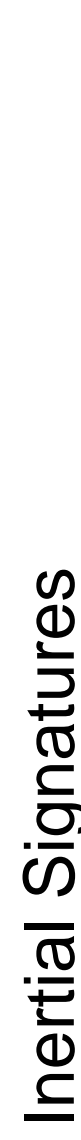

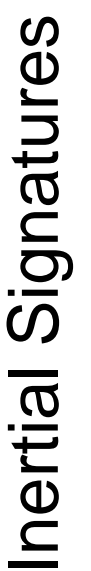

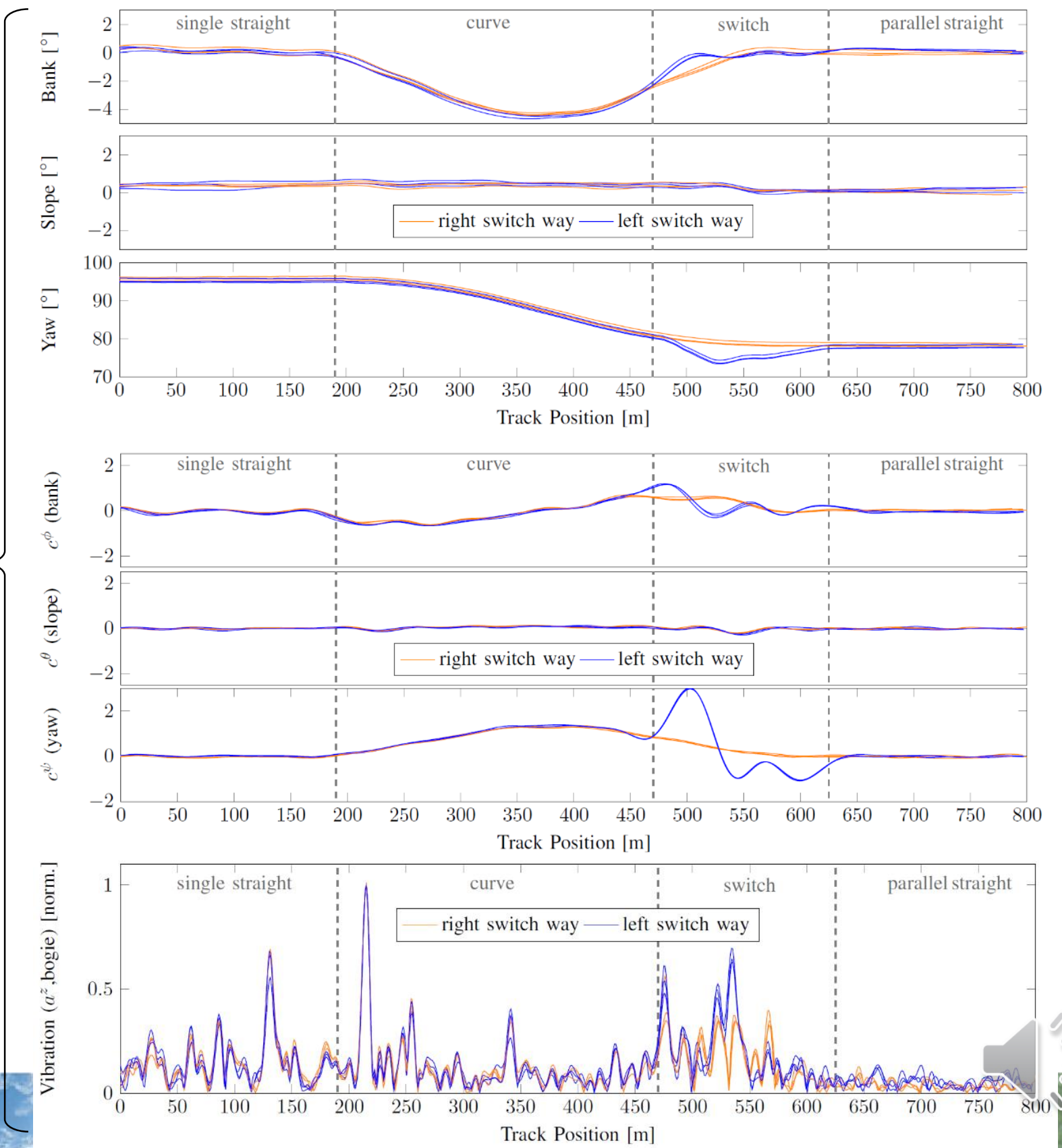




\section{IMU Measurement Methods}

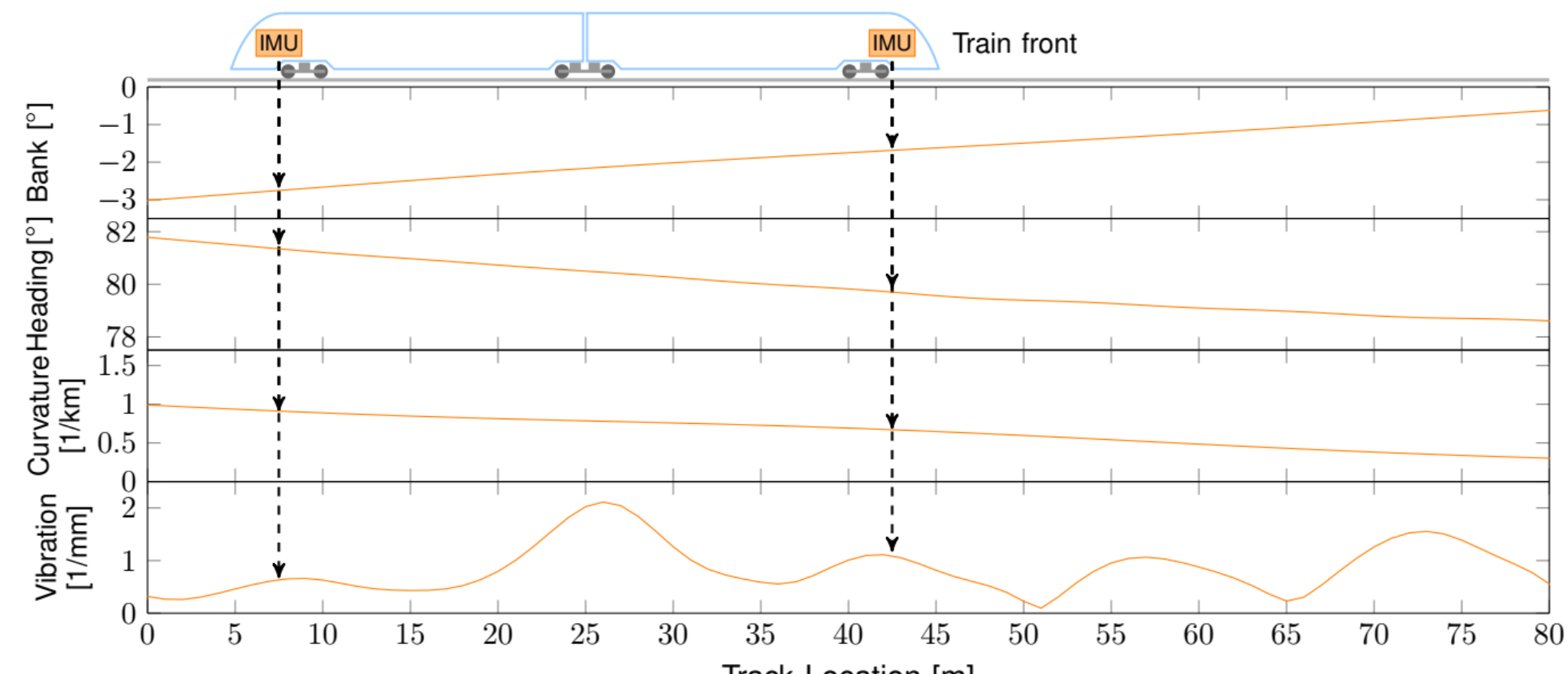

\section{Train Speed, Relative over Time}

Along-track acceleration, IMU integration over time

Vibration speed signature, comparison of speed dependent patterns

Vibration signature comparison 2x IMU with known distance between

\section{Relative Train Localization} Inertial signature relative on $2 x \mathrm{IMU}$ tracks
Absolute Train Localization Inertial signature (attitude, IMU, map curvature, vibration) 


\section{Magnetometer}

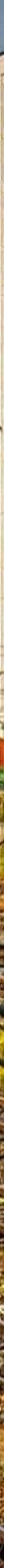




\section{Magnetometer: Magnetic Signature,} 1st Run
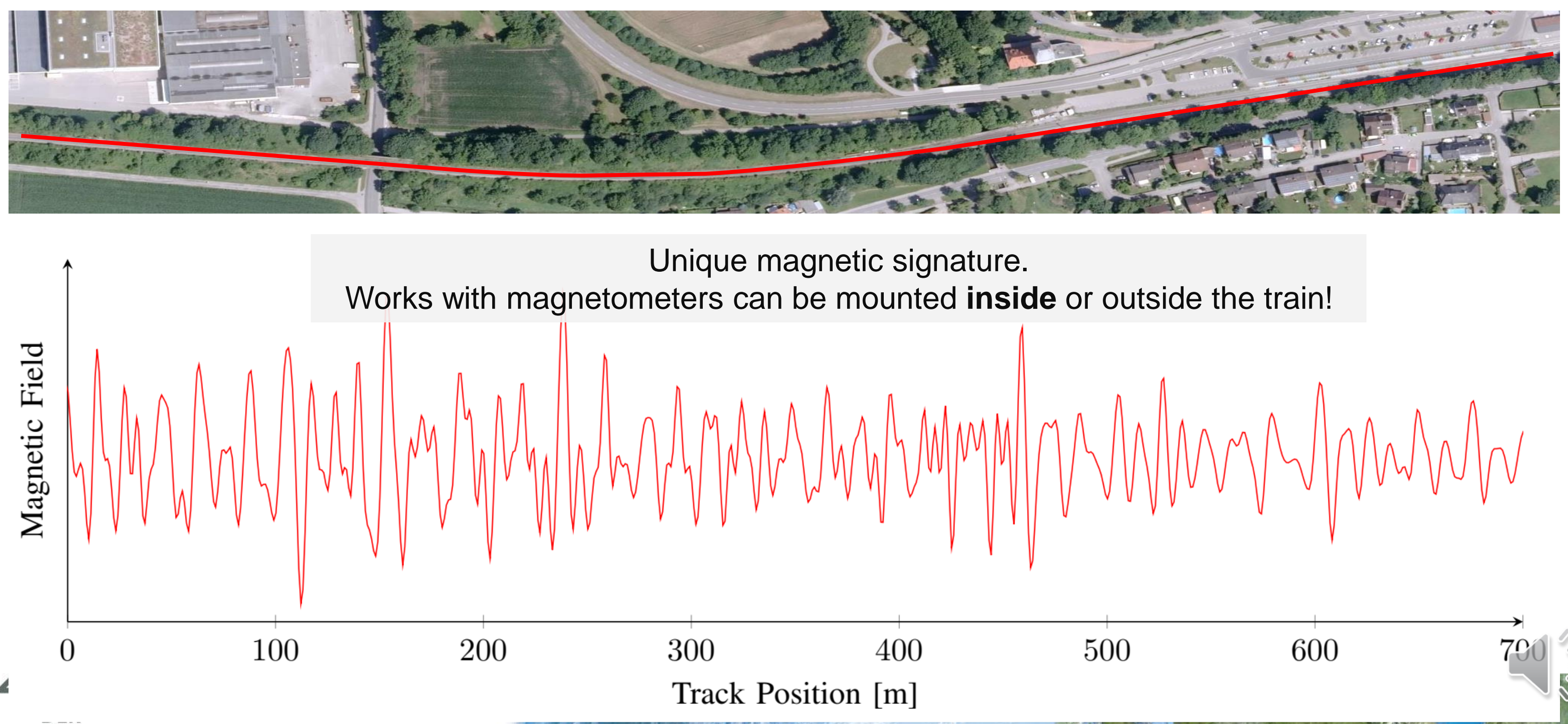


\section{Magnetometer: Magnetic Signature} 1st Run + 2nd Run
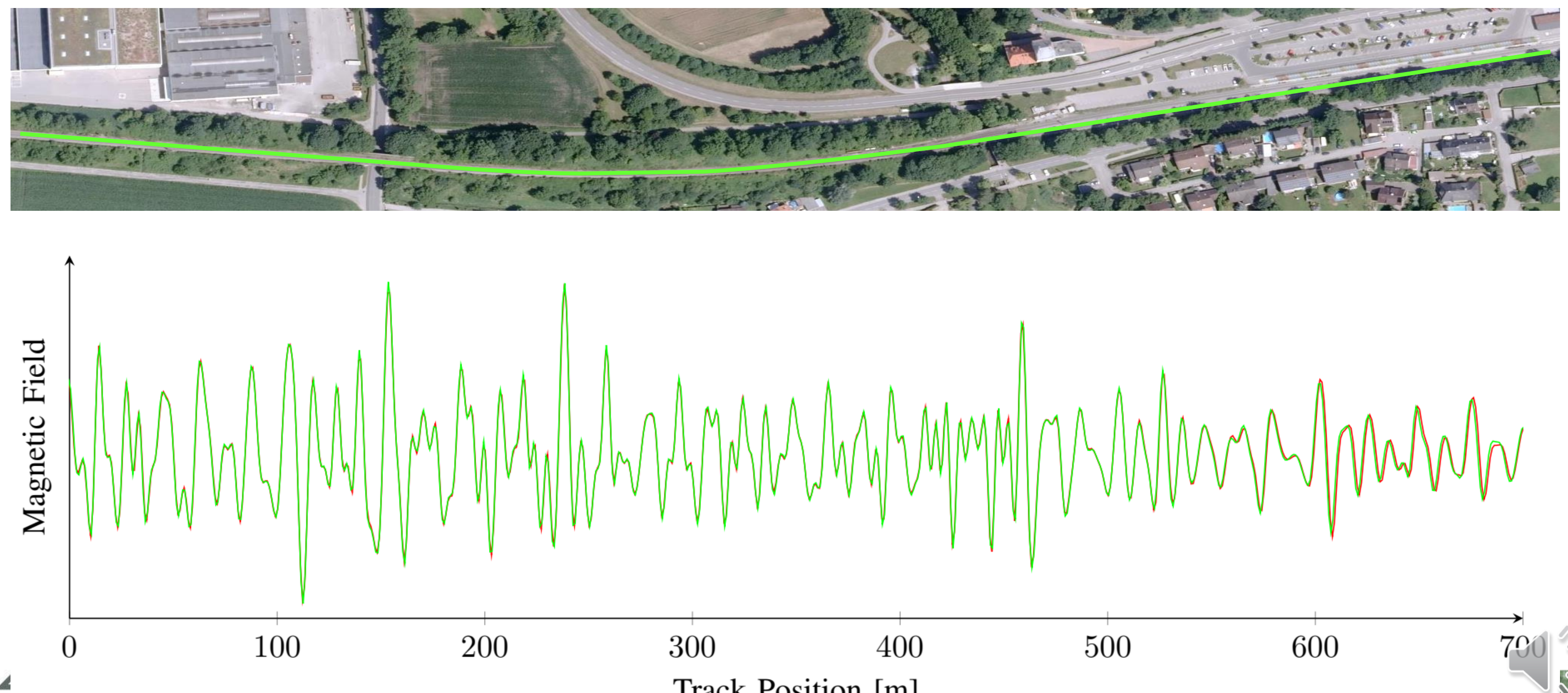

Track Position [m] 


\section{Magnetometer: Magnetic Signature 1st Run + 2nd Run + 3rd Run}
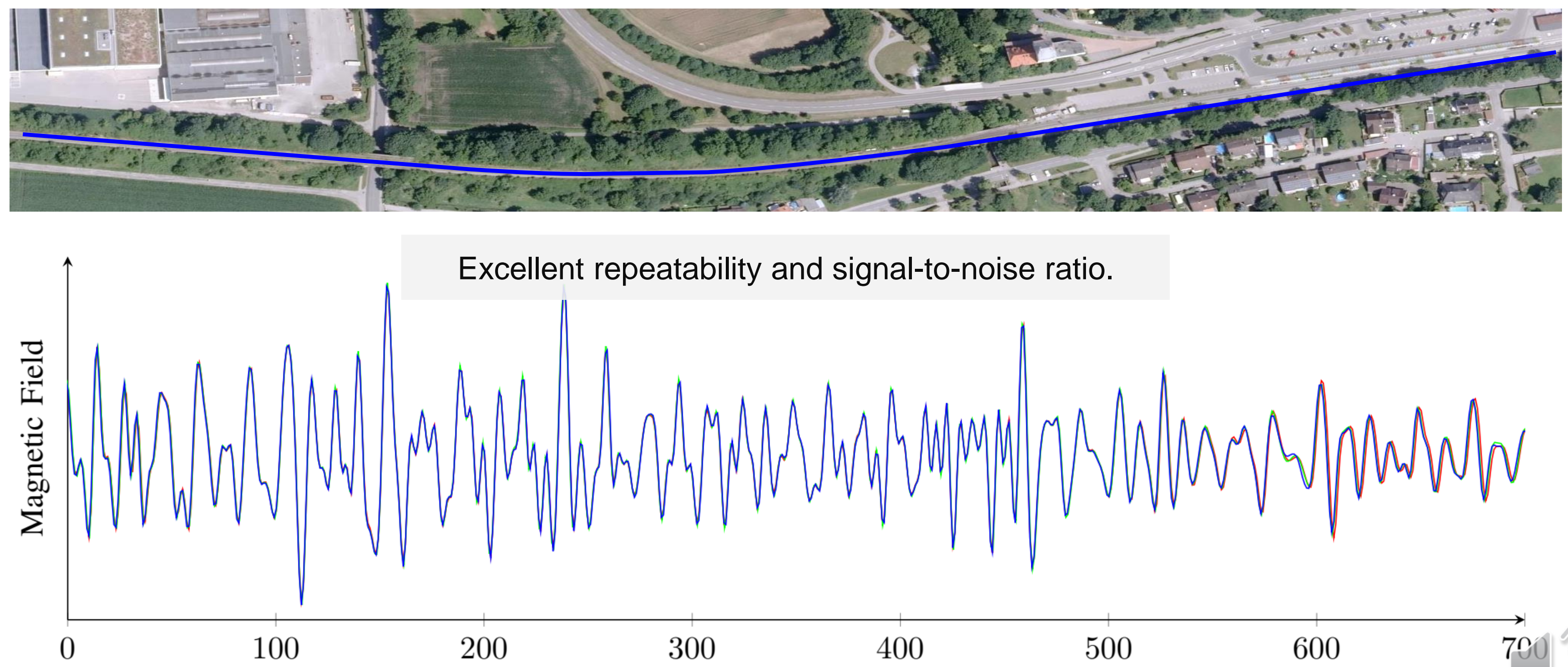

Excellent repeatability and signal-to-noise ratio.

0

100

200

300

400

500

600

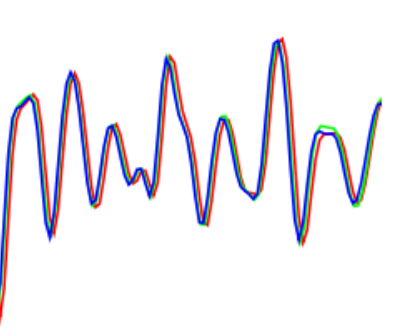

Track Position [m] 


\section{Magnetometer: Magnetic Signature 6 rain runs}
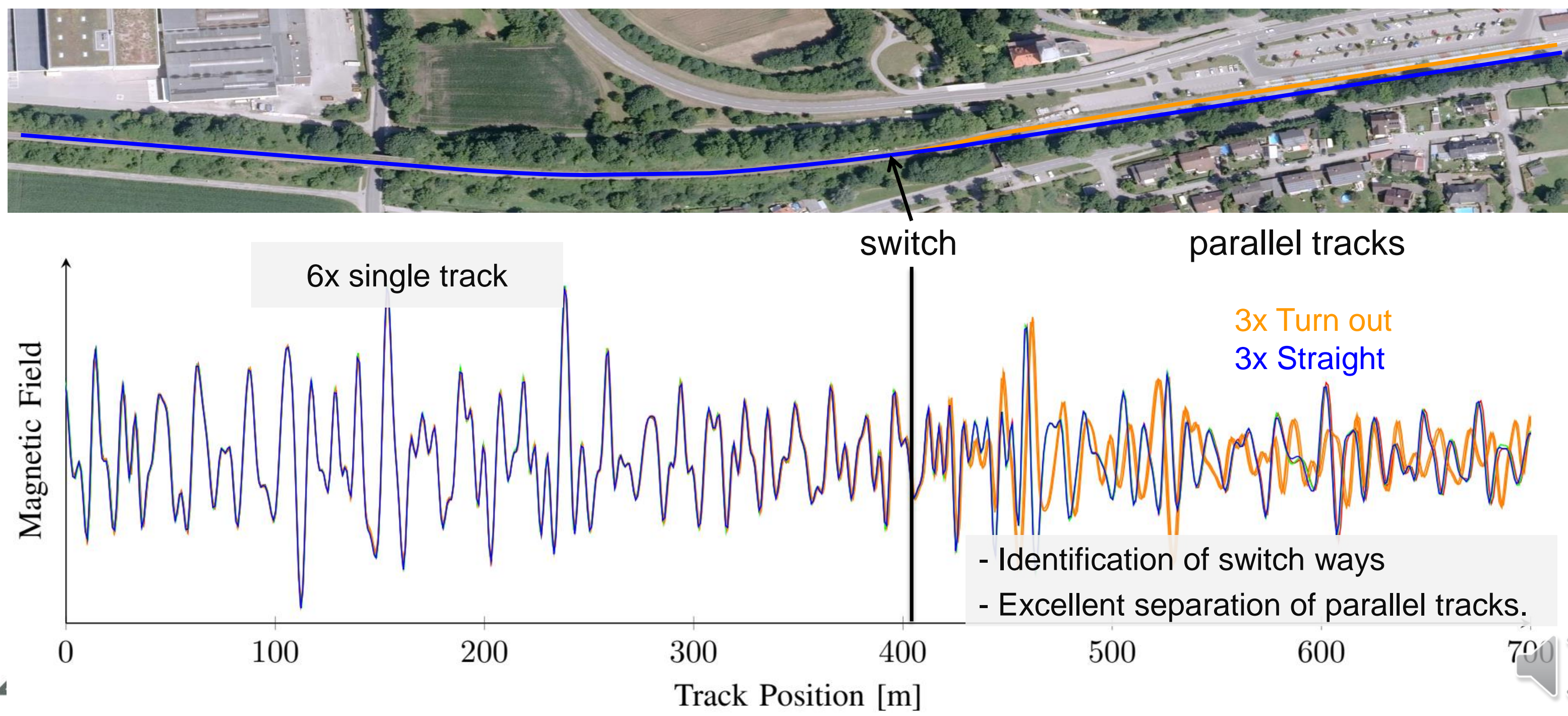

\section{parallel tracks}

3x Turn out

$3 x$ Straight

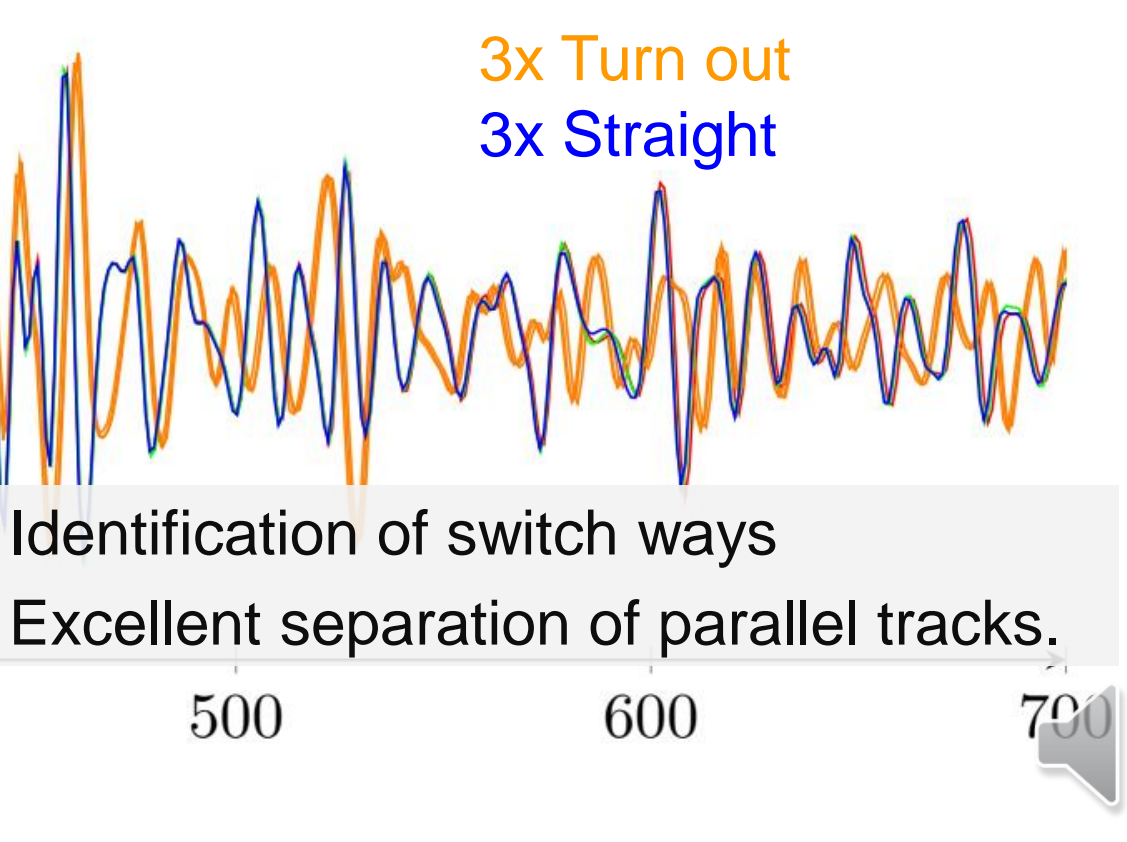




\section{Magnetic Measurement Methods}

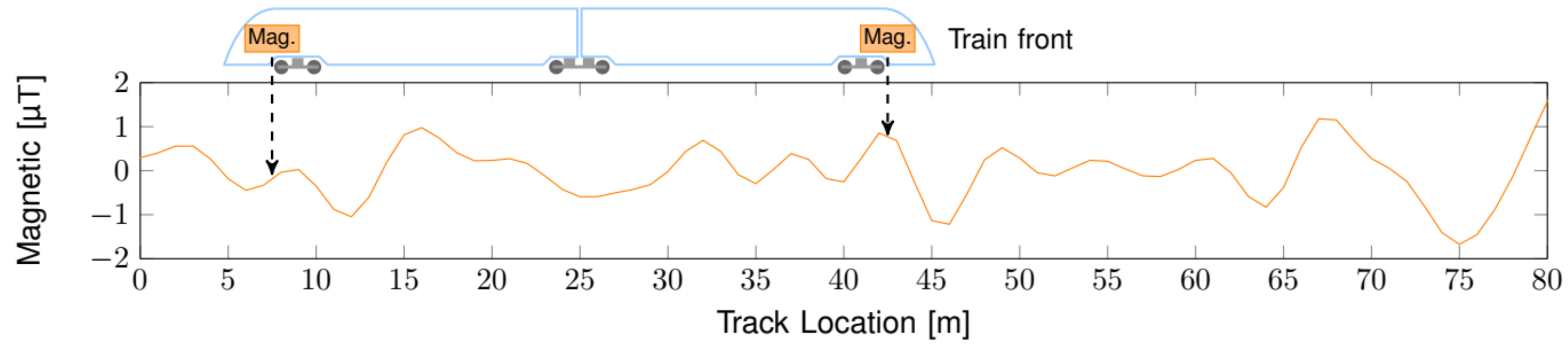

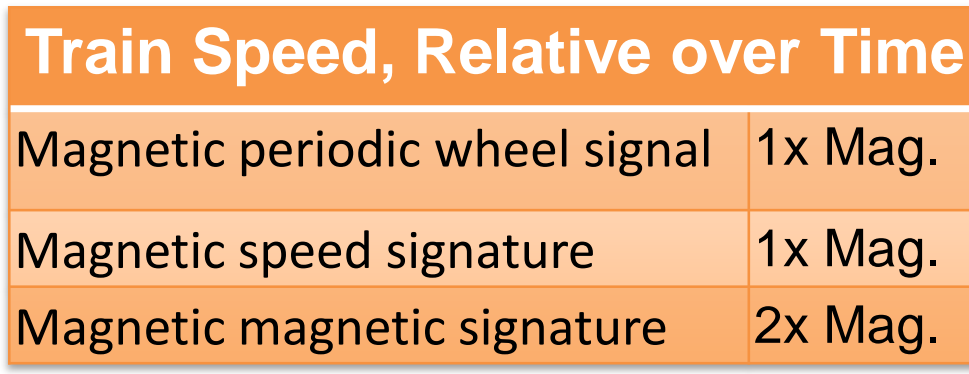

\section{Relative Train Localization} Magnetic signature relative $2 x$ Mag. on tracks (Odometry)

\section{Absolute Train Localization} Magnetic signature absolute Mag., map on tracks (odometry) INS/magnetic/map Mag, IMU (odometry)

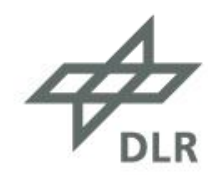




\section{Terrestrial Radio Ranging Measurement Methods}
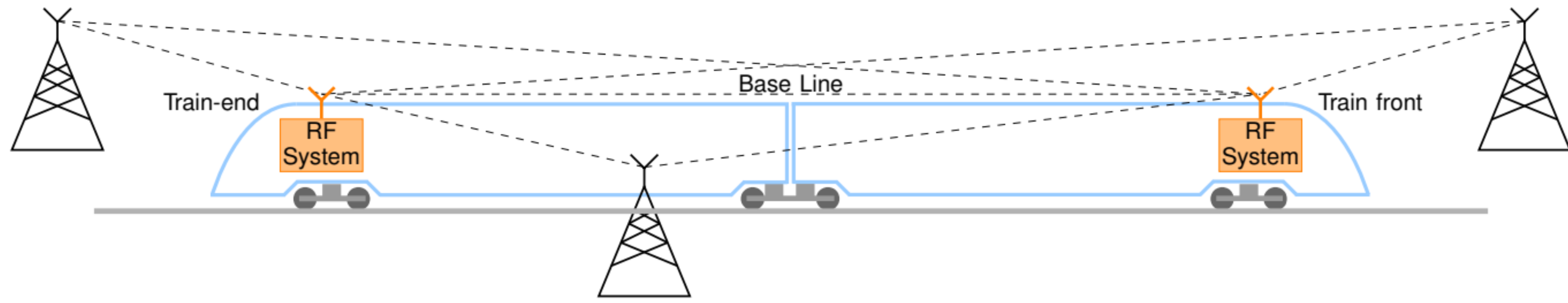

- Measurements: Ranges (Round-trip Delays), Doppler

Train Speed, Relative over Time

Cellular RF system, Doppler of mobile base station to mobile terminal terminal

\section{Relative Train Localization} Radio ranging on train $2 x \mathrm{RF}$ trans.

Cellular base station, $2 x$ mobile difference

\begin{tabular}{|l|l|}
\hline \multicolumn{2}{|c|}{ Absolute Train Localization } \\
$\begin{array}{ll}\text { Cellular base station } \\
\text { ranging, map-matched } \\
\text { position, loosely coupled }\end{array}$ & $\begin{array}{l}\text { mobile } \\
\text { terminal, map }\end{array}$ \\
$\begin{array}{l}\text { Cellular base station } \\
\text { ranging, tightly coupled }\end{array}$ & $\begin{array}{l}\text { mobile } \\
\text { terminal, map }\end{array}$ \\
\hline
\end{tabular}

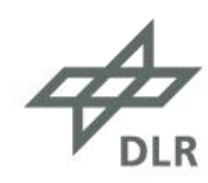




\section{Current \& Future Work}

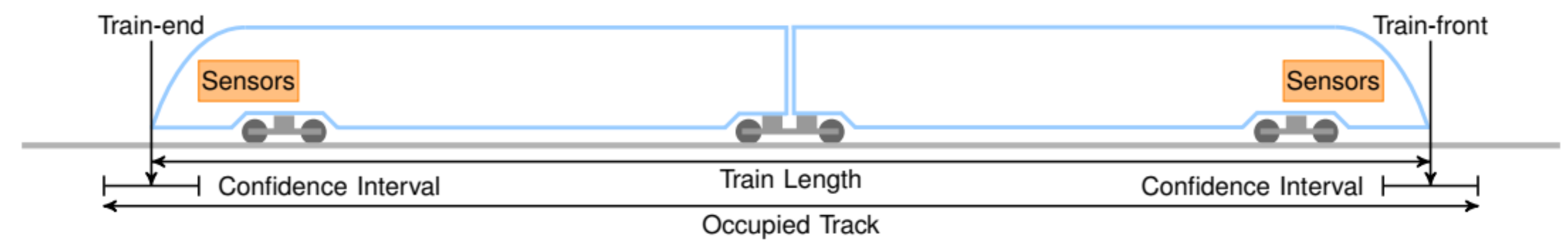

- Measurement methods from GNSS, IMU, magnetometer and terrestrial radio ranging.

- Current \& future research focuses on:

- Localization algorithms based on a digital track map with INS/GNSS, inertial and magnetic signatures for a reliable train speed, train location and train integrity monitoring with highest availability in all environments

- Safe train localization with navigation integrity methods for railways with advanced sensor models 


\section{Measurement Methods for Train Localization with Onboard Sensors}

Oliver Heirich
Benjamin Siebler
Stephan

Sand
Andreas Lehner
Omar

García Crespillo
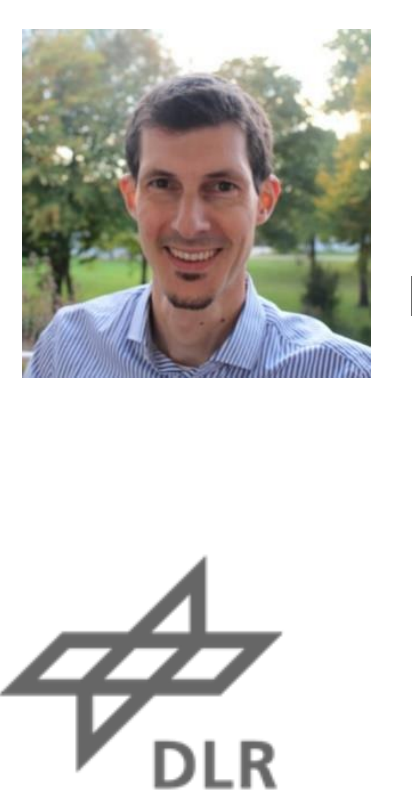

Q\&A Session: D2 Rail Navigation, Day 1 (Monday, 23.11) 19:00-19:20 CET

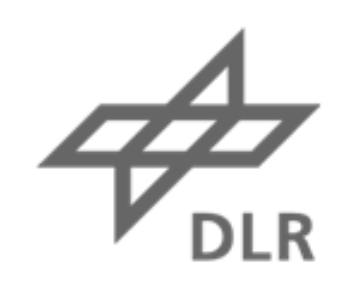

Dr.-Ing. Oliver Heirich

oliver.heirich@dlr.de

DLR - German Aerospace Center Institute of Communications and Navigation Oberpfaffenhofen, Germany

Links to all our publicatio
elib.dlr.de Knowledge for Tôm

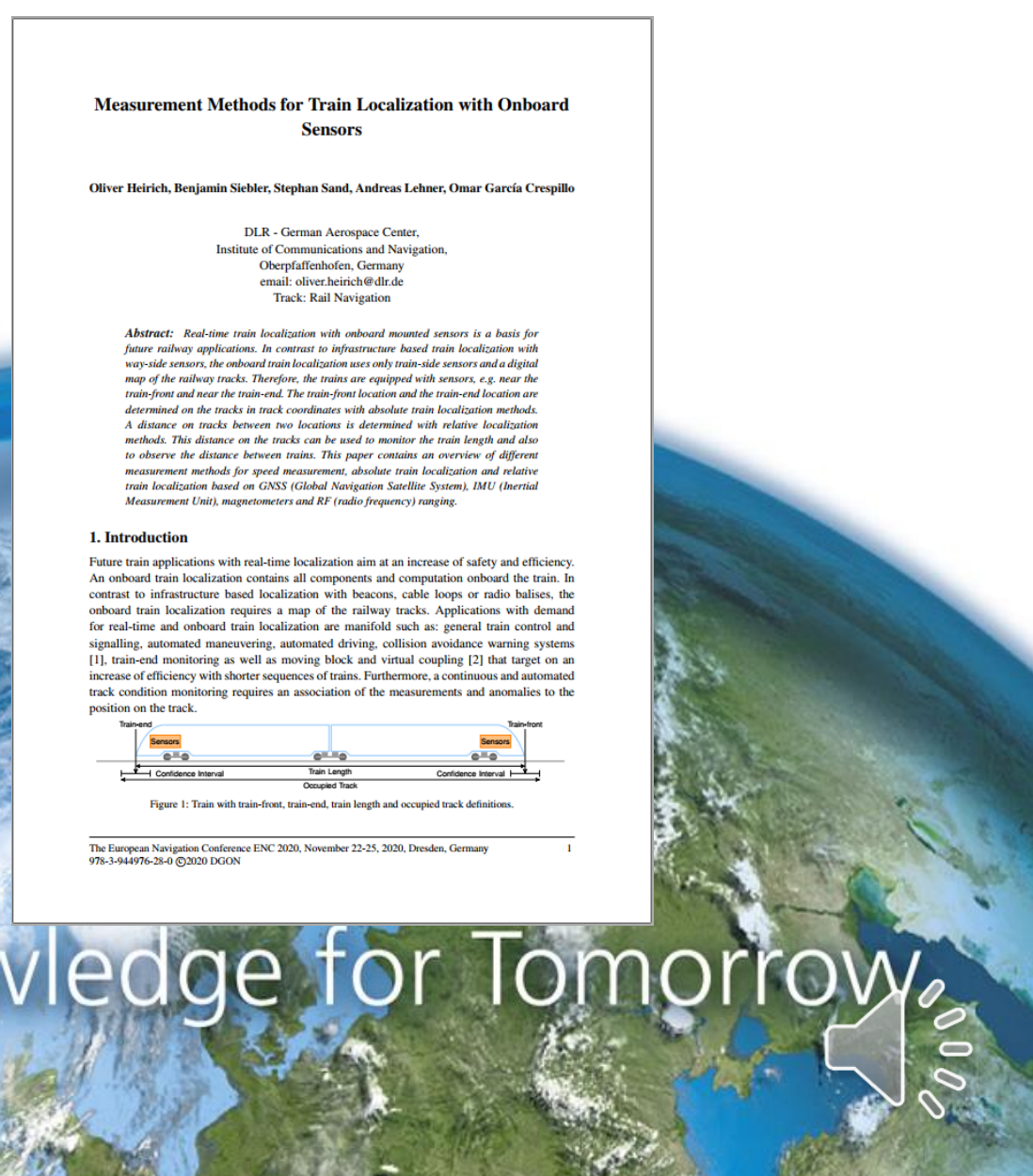

